\title{
Visual Tracking and Following of a Quadrocopter by another Quadrocopter
}

\author{
Karl E. Wenzel, Andreas Masselli and Andreas Zell
}

\begin{abstract}
We present a follow-the-leader scenario with a system of two small low-cost quadrocopters of different types and configurations.

The leader is a Parrot AR.Drone which is controlled by an iPad App utilizing the visual odometry provided by the quadrocopter and pilots it autonomously. The follower is an Asctec Hummingbird which is controlled by an onboard 8-bit microcontroller. Neither communication nor external sensors are required. A custom-built pan/tilt unit and the camera of a Nintendo Wii remote tracks a pattern of infrared lights and allows for online pose estimation. A base station allows for monitoring the behavior but is not required for autonomous flights.

Our efficient solution of the perspective-3-point problem allows for estimating the pose of the camera relative to the pattern in six degrees of freedom at a high frequency on the microcontroller. The presented experiments include a scenario in which the follower follows the leader with a constant distance of two meters flying different shapes in narrow, GPS-denied indoor environment.
\end{abstract}

\section{INTRODUCTION}

In recent years much research has been done to provide full autonomy for unmanned aerial vehicles (UAVs), including self-localization, obstacle avoidance and automated landing maneuvers. In this paper the interaction of two quadrocopters during flight is examined which is necessary for multiple UAVs to collaborate. Even heterogeneous constellations are conceivable, with one or more UAVs being controlled by a human operator. To achieve interaction in the air, a UAV has to be able to perceive its fellows and align itself with respect to them. For our examination we consider a follow-the-leader-scenario, where we focus on the abilities of a quadrocopter (QC) to follow a second one and maintain a constant position and orientation relative to the leader during flight (Fig. 1). The abilities of the follower have been shown in a former publication [13], in which the QC tracked a moving carrier ground vehicle. The leader in current experiments is no longer on the ground, but also in the air. This task is more challenging, since now the object being tracked moves less predictably and tends to more rotational movement. Larger errors are induced which have to be balanced out by the flight controller of the follower. Furthermore, the leader produces wind turbulences that interfere with the follower, since both UAVs fly close to each other. The task is solved using only onboard processing on the follower. No wireless communication is used to aid

Karl E. Wenzel and Andreas Masselli are PhD students with the Chair of Cognitive Systems, headed by Professor Andreas Zell, in the Department of Computer Science, University of Tübingen, Sand 1, 72076 Tübingen, Germany, Phone: +49 7071 76455, Fax: +49 7071 5091, Email: \{karl.e.wenzel, andreas.masselli, andreas.zell\} @uni-tuebingen. de the follower, neither with the leader nor with a base station. Moreover, the goal was to use only low-cost hardware. Since the experiments were done indoors, the positioning had to be accurate.

Quadrocopters are helicopters with four independent, fixed rotors. By varying the speeds of the four corresponding motors, the aircraft can move in six degrees of freedom. Bouabdallah et al. [2] analyze the advantages of quadrotors and describe their dynamic model.

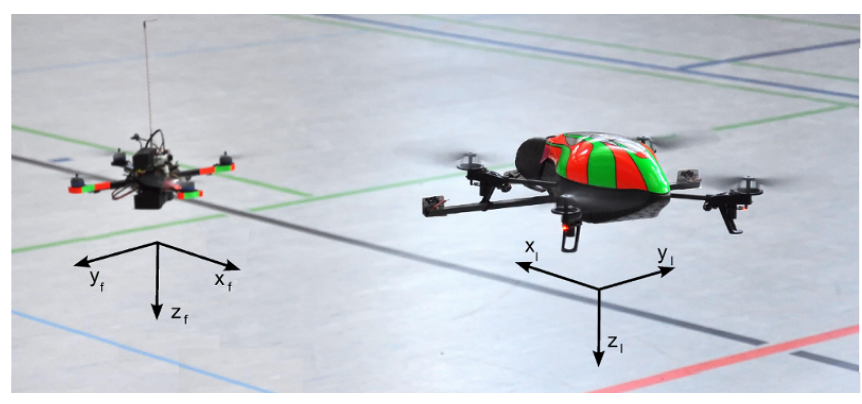

Fig. 1. Leader (Parrot AR.Drone, right) and follower (Asctec Hummingbird, left) with relative position and coordinate systems

\section{RELATED WORK}

Following a leader is a well-known research topic for ground robots. In 2002 Aveek et al. introduced a framework for cooperative control of a group of nonholonomic mobile robots where the lead robot's motion defines the bulk motion of the group [3]. Orqueda et al. describe a visionbased decentralized controller which only requires knowledge of the leader-follower relative distance and bearing [11]. Mellinger et al. show that QCs are capable of aggressive autonomous maneuvers [9] and that QCs can autonomously work together in a team grasping and transporting more weight than a single QC could carry [10]. Beard [1] present how multiple UAV could cooperatively search a region with unknown opportunities and hazards. These projects, however, consider equal configurations of the UAVs and assume the knowledge of each pose of the UAVs. A Vicon motion capture system was used for indoor experiments, resulting in a limited working area within the sight of the cameras. Yun et al. present a leader-follower system for unmanned helicopters [14], but their results are produced with a virtual leader and a real follower. Other projects involve quasi-stationary flight [5] and vertical landing control [6]. The ground station does the calculation in these projects, which leads to restrictions in autonomy. Our setup allows for starting, landing and hovering precisely with low-cost onboard sensors and artificial beacons [12], [13]. 


\section{THE FLYING LEADER}

In our scenario, the leader quadrocopter $(\mathrm{QC})$ provides basic autonomy such as stationary hovering and onboard odometry. This allows for self-directed flights and thus for guiding a follower to a desired location. The leader is capable of flying on predefined paths at a given speed and is tracked by the follower via an attached IR pattern.

\section{A. Hardware}

The toy helicopter AR.Drone by Parrot has been available since summer 2010. We applied minor modifications to the original hardware by attaching a pattern of infrared lightemitting diodes (LEDs) to provide the detection for the follower. (Fig. 2)

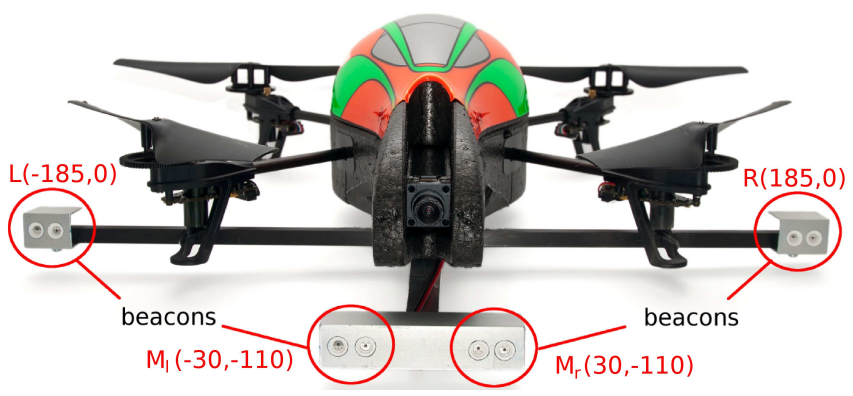

Fig. 2. The AR.Drone with a pattern of eight LEDs, creating four blobs of infrared light. The units of the position of the blobs is given in millimeters.

1) Quadrocopter: The total weight of the QC is $380 \mathrm{~g}$, the diameter is $55 \mathrm{~cm}$. It is controlled via $\mathrm{WiFi}$, either with an iPhone-compatible device, or a Linux computer. The aircraft comes with a front camera and a vertical camera, an ultrasonic sensor, MEMS gyroscopes, acceleration sensors, brushless motors and an ARM9 embedded computer with $468 \mathrm{MHz}$ and $128 \mathrm{MB}$ RAM. The vertical camera and the ultrasonic sensor provide onboard odometry, which also ensures stable hovering when no commands are sent.

2) Pattern: The pattern to be tracked by the follower consists of four pairs of LEDs. All LEDs are within the extent of the drone, lie in one plane and form a symmetrical pattern.

Although three LEDs would suffice for pose estimation, we decided to add a fourth LED to rate the quality of the estimate. Since three light sources always yield a possible solution for the pose estimate, by calculating the reprojection error the fourth LED can be used as a check that it is the actual pattern the follower is seeing. The pattern is recognizable as long as no LEDs are hidden or overlapping. The current setup allows for an offset of about $45^{\circ}$ in any direction up to a distance of $3 \mathrm{~m}$.

\section{B. Software}

A Linux system runs on the onboard computer of the AR.Drone. We upgraded an iPad compatible Objective$\mathrm{C}$ application to autonomously fly along waypoints. The AR.Drone provides pose information relative to the starting point but depends on visual features on the ground. With each new video frame, a new pose estimate is available. The WiFi signal strength can influence the update rate. In our experiments we achieve about $20 \mathrm{~Hz}$. An additional screen in our application allows for choosing the shape parameters of the flight path and to toggle logging and autonomous flight.

Three independent PID controllers regulate the values for roll, pitch, and yaw. The drone keeps its current height by use of the onboard controller if no thrust commands are sent. One can choose the shape and size of the flight path as well as the leader's target speed. With every new pose estimate, the error is calculated and a new waypoint and control data are generated.

A special gesture is performed by the leader when switching to autonomous flight: The drone pitches (nods) three times before leaving the stationary hover position. This helps finding the start of autonomous flights in the log file of the follower. Otherwise synchronization of the experimental data of both quadrocopters would be difficult.

\section{THE FLYING FOLLOWER}

In our scenario, the follower only knows its relative position to the leader and the distance to the ground. Thus it can hardly differentiate between movements of its own and the leader. The follower, however, distinguishes between orientation changes of itself and the leader by use of the pan/tilt unit of the onboard camera.

\section{A. Hardware}

Our follower platform is an AscTec Hummingbird Autopilot, a well known platform in current quadrocopter research. Compared to the AR.Drone it's flight behavior is much more agile but provides less autonomy. Figure 3 shows the configuration used for the experiments, employing a special infrared camera, a small pan/tilt unit and two infrared sensors to measure the distance to the ground.

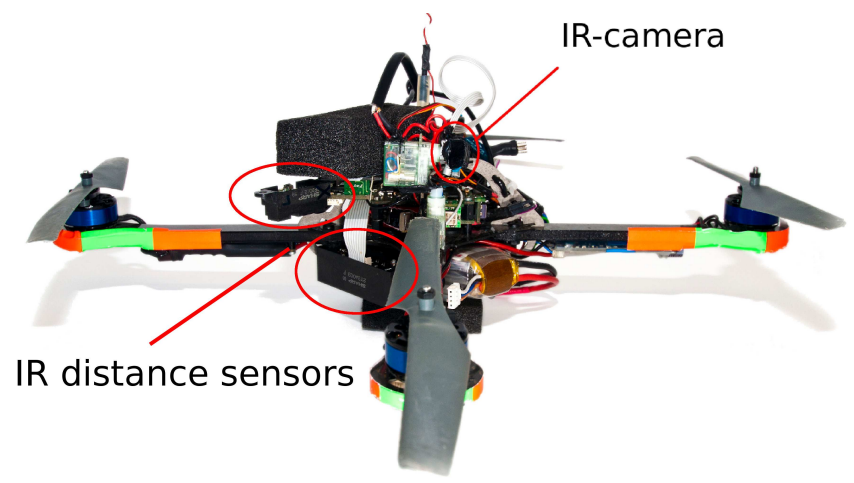

Fig. 3. The customized Hummingbird quadrocopter

1) Quadrocopter: The Hummingbird Autopilot provides two $60 \mathrm{MHz} 32$ bit ARM microcontrollers with inertial measurement unit, a magnetometer, and a pressure sensor. The $\mathrm{QC}$ is $53 \mathrm{~cm}$ in diameter at a total weight of around $550 \mathrm{~g}$. An additional onboard ATMega 1284P microcontroller with a ZigBee-Pro serial radio transmission module is attached to 
the serial port of the quadrocopter and allows for communication with the base station over up to $1 \mathrm{~km}$. The microcontroller also provides digital and analog input/output ports and an $\mathrm{I}^{2} \mathrm{C}$ bus to connect additional hardware. Two infrared distance sensors, one for short range (Sharp GP2D12) and on for long range (Sharp GP2Y3A003), were additionally connected to measure the distance to the ground.

2) Wii Remote Camera: The controller of the Nintendo Wii game console, informally known as Wiimote, includes an infrared camera, which communicates via $\mathrm{I}^{2} \mathrm{C}$ and provides the pixel positions of up to four infrared light sources in the frame. In our experiments $200 \mathrm{~Hz}$ and more were possible. Its small size of $8 \times 8 \times 5 \mathrm{~mm}^{3}$ and its weight make the camera an ideal onboard sensor for small UAVs, and the ready to use data disburdens online object recognition. The resolution of $1024 \times 768$ pixels, achieved by eight times subpixel analysis, as well as additional information such as intensity and size of the blobs support sufficient recognition abilities.

3) Pan/Tilt Unit: Two miniature servo motors are used as a minimalistic, lightweight pan/tilt unit. The unit enlarges the range of operation since the camera can be directed to the IR-pattern even if the pattern is not directly in front of the follower. We connected the potentiometer of each servo motor to an analog input of the microcontroller and defined a calibration function to estimate the current servo angle from the voltage measurements. This gives us much more precise and faster results than estimating the servo position over time by means of the PWM signal. Experiments have shown an accuracy of around $1^{\circ}$. Knowing the exact current angle allows for fast and efficient centering of the pattern in the camera frame and ensures exact pose estimation of the follower relative to the leader.

\section{B. Software}

No ground station is required for autonomous flights since all processing is done on an onboard microcontroller. However, a base station is used for monitoring, parameter tuning, and logging. In each cycle of the main control loop the pose relative to the leader is estimated in six degrees of freedom (DOF) using the IR camera. The height over ground is estimated by the IR distance sensors. Then, four independent, classical proportional-integral-derivative (PID) controllers pilot the follower via the serial interface to maintain a constant $\mathrm{x}, \mathrm{y}$-position and orientation relative to the leader as well as a constant height over ground. Currently, an update rate of $25 \mathrm{~Hz}$ is achieved, limited by the amount of data which is sent to the base station.

1) Pilot: A high-level finite state-machine machine guides the follower. By utilizing the two IR sensors autonomous departure, maintaining a desired height, and gentle landing is possible, even without sight of the pattern. A switch on the remote control causes the motors to start, takeoff will be initiated and a constant rise of the desired height up to $1 \mathrm{~m}$ follows. The default desired position is fixed $2 \mathrm{~m}$ behind the leader and could be changed by an operator. Landing is either initiated via the base station, or if the pattern is lost for more than 20 cycles (almost one second).
2) Pose estimation: Because of the lack of odometry, the pose of the follower cannot be calculated in world coordinates. Only the pose relative to the leader can be estimated in $6 \mathrm{DOF}$ by solving the perspective-three-point problem assigned to our configuration. To obtain more information about the pose in the world we integrate the data from the infrared distance sensors in the estimation. The height over ground is estimated by the IR sensors, depending on the sensor range, and smoothed by a Kalman filter.

Since the follower should keep the same yaw angle as the leader, the error of the yaw angle $\psi$ is the difference in orientation of both QCs.

\section{PnP}

The problem of calculating the relative pose from a camera to a set of markers with known positions is known as the perspective-n-point problem [4]. To solve the problem we developed our own method to allow real-time processing even on a microcontroller. By using several constraints given by the experimental setup, namely the restricted operating range and the symmetry of the pattern, we could provide fast evaluation of the pose. We compared our approach to the one presented in [7]. While showing a comparable accuracy, our method runs about 20 times faster, yet providing only one solution and being restricted to the symmetric patterns, which is appropriate for our application. The pose estimation method can be run with $40 \mathrm{~Hz}$ on the ATMega microcontroller, and has proven to be robust and accurate enough for our experimental setup (see Section V for details). The same method was implemented for a Gumstix processor which processes the image and controls a quadrocopter at $60 \mathrm{~Hz}$. For details see [8].

1) Spot Identification: We use four IR spots as control points, whose coordinates are given in the coordinate system of the leader. The Wii Remote camera directly returns the image coordinates of these points once they are seen by the camera. It has to be clarified which spot in the image corresponds to which control point in the leader's pattern. Since it can be assumed that the quadrocopter is not in an upside-down position or behind the pattern, the spots can simply be sorted by their $x$ coordinate to identify them as left, middle-left, middle-right and right.

2) Solving the PnP Problem: Given four points of the leader pattern $L, M_{l}, M_{r}, R$ in the leader's coordinate system and four corresponding points in the image plane $L_{i}, M_{l i}$, $M_{r i}, R_{i}$ in pixel coordinates.

First, we consider a virtual fifth point $M$, which is the center of $M_{l}$ and $M_{r}$. We estimate its projection $M_{i} \approx$ $\frac{M_{l i}+M_{r i}}{2}$ onto the image plane. Then, we use $L, M, R$ and their corresponding image points to obtain the camera position $P$. If only three control points are used, a maximum of four locations of $P$ are possible [4]. In our case the operating range is restricted, so the solutions can easily be narrowed down to the one which represents the actual position of $P$. The control points $M_{l}$ and $M_{r}$ together with $M_{l i}$ and $M_{r i}$ are used afterwards to rate the position estimate by calculating the reprojection error. 
By assuming a standard pinhole camera model, whose focal length $f$ was experimentally determined for the IR camera, we expand the image coordinates into $3 \mathrm{D}$ vectors setting $z_{L_{i}}=z_{M_{i}}=z_{R_{i}}=f$. We calculate a foot $M_{i}^{\prime}$ of $M_{i}$ onto the plane through $L_{i}, R_{i}$ and origin $O_{i}$ of the camera coordinate system. Then we define three angles $\alpha=\angle\left(M_{i}^{\prime}, R_{i}\right), \beta=\angle\left(L_{i}, M_{i}^{\prime}\right)$ and $\gamma=\angle\left(M_{i}^{\prime}, M_{i}\right)$ with $\angle(A, B)=\arccos \frac{A \cdot B}{|A| \cdot|B|}$.

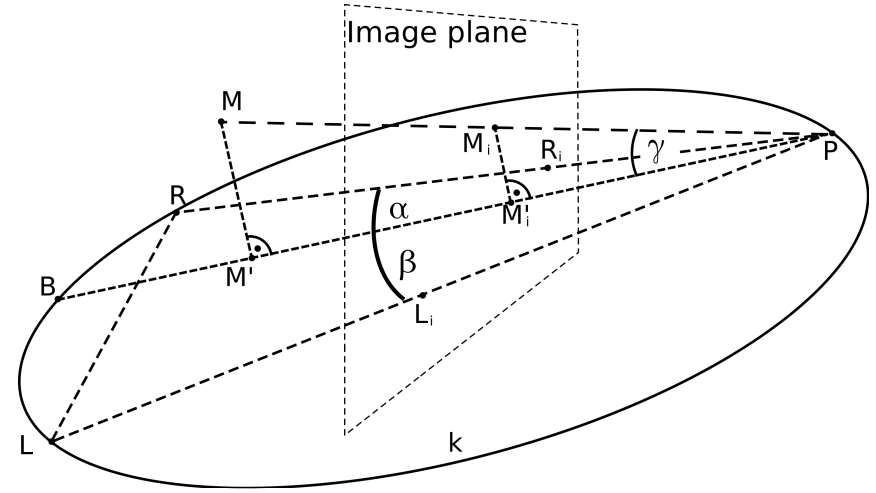

Fig. 4. Geometric representation of the plane $E$, where the points $L, R$ and $P$ are located, the angles $\alpha, \beta, \gamma$, and the image plane.

Consider the plane $E$ through the points $L, R$ and $P$. Define a right-handed Cartesian coordinate system with $M_{L R}=\frac{L+R}{2}$ as origin, $\mathrm{y}$-axis through $R$ and $\mathrm{x}$-axis lying in $E$ (Fig. 4). Let $M^{\prime}$ be the foot of $M$ onto the plane $E$. Note that $M_{i}^{\prime}$ equals the projection of $M^{\prime}$ onto the image plane, which yields $\angle\left(M^{\prime}, R\right)=\alpha$. We know that $M$ lies on a circle $l$ with radius $r=d(M, O)=110 \mathrm{~mm}$. This means that $M^{\prime}$ lies on the projection of the circle, which is a segment between two extrema $M_{\min }=(-r, 0)$ and $M_{\max }=(r, 0)$. We know from the inscribed angle theorem that $P$ lies on a circle $k$ with center $C=\left(\frac{y_{R}}{\tan (\alpha+\beta)}, 0\right)$. Using the inscribed angle theorem again, we can construct a point $B$ on $k$ such that the angle between the segments $P B$ and $P R$ equals $\alpha$. $B$ is constructed by rotating $R$ around $C$ by $2 \alpha$. Note that $B$ is constant for all possible positions of $P$ on $k$.

Since $M^{\prime}$ lies on the segment $P B$, we can now describe $P$ as a function of $M^{\prime}$, or, since $y_{M^{\prime}}=0$, as a function of $x_{M^{\prime}}$. The task is to find $x_{M^{\prime}}$. To achieve this, we look at two things: First, the distance of $M$ to $E$, which can be calculated by

$$
d(M, E)=d\left(M, M^{\prime}\right)=\sqrt{r^{2}-x_{M^{\prime}}^{2}}
$$

since $M$ lies on $l$.

Second, we calculate $h=a m$, with $a$ being the length of segment $P M^{\prime}$ and $m=\tan \gamma$ being the slope of $P M$ in $z$ direction. The following equation has to hold:

$$
d(M, E)=h
$$

Squaring and plugging in the values lead to:

$$
r^{2}-x_{M^{\prime}}^{2}=m^{2} \cdot a^{2}
$$

Further expansion yields the quartic polynomial

$$
c_{4} x^{4}+c_{3} x^{3}+c_{2} x^{2}+c_{1} x+c_{0}=0
$$

with $x=x_{M^{\prime}}-x_{C}$ and coefficients

$$
\begin{aligned}
& c_{4}=m^{2}+1 \\
& c_{3}=2\left(x_{C}-x_{B}\right) \\
& c_{2}=v+\left(1-2 x_{C}^{2}\right) B^{2}-4 x_{C} x_{B} \\
& c_{1}=2\left(x_{C} B^{2}-v x_{B}\right) \\
& c_{0}=v B^{2}+m^{2}\left(B^{2}\right)^{2},
\end{aligned}
$$

with $v=x_{C}^{2}-r^{2}$ and $B^{2}=x_{B}^{2}+y_{B}^{2}$.

Since we can assume that the pattern is upright and facing the follower, the valid solution is the one with $M$ being closest to the camera, i.e., the largest root of (1) belongs to the valid solution. This root is found using four iterations of Newton's method, starting with $x_{0}=x_{M_{\max }}-x_{C}$.

Once the root $x_{r}$ is calculated, $P$ can be determined by intersecting the line through $B$ and $M^{\prime}=\left(x_{r}+x_{C}, 0\right)$ with circle $k$ and transforming its coordinates into the leader coordinate system.

Singularities appear only if the camera is on the circle defined by $L, M, R$, which does not happen in our scenario.

3) Confidence Estimation: A confidence of the pose estimation is calculated using the reprojection error. The current pose is used to map all four control points $L, M_{l}, M_{r}$ and $R$ into camera space. Then we project the points using the pinhole camera model from above. The projected points are finally compared with the actual image points returned from the camera using the sum of Euclidean distances. The distance error is converted into a probability using a Gaussian with an empirically determined standard deviation of 11 pixels. A perfect shape results in the maximum confidence value of $100 \%$. The confidence value is used by Kalman filters, which refine short periods where the pattern is invisible as well as outliers of the pose estimate for $x, y$, $z$ and yaw.

Knowing the camera position $P$, the follower's position can be directly calculated using the transformation matrix of the pan/tilt unit and the two servo angles.

4) PID Controller: Four independent PID controllers pilot the follower. The only modification to the controllers described in [13] are filters which make the loop more sensitive if the quadrocopter is near the desired position and less if the error becomes larger.

\section{EXPERIMENTS}

We flew multiple times with a single and with both quadrocopters at the same time to test the performance of our system. Videos can be found in our YouTube channel ${ }^{1}$.

\section{A. The Task}

The follower had to keep a constant position $2 \mathrm{~m}$ behind the pattern. Since the follower cannot differentiate between its own movements and those of the leader carrying the pattern, the leader could fly any path resulting in the other quadrocopter following. Each orientation change of the

\footnotetext{
${ }^{1}$ http://www.youtube.com/user/ZellTuebingen
} 
leader leads to a change in the followers desired position. This ensures that the sight on the pattern is kept.

Our experience shows, that the leader was influenced by the wind produced by the follower and we tried to reduce the effect by choosing a distance between both aircrafts and a height where the effect is acceptable. First experiments involve only the Hummingbird and demonstrate the performance without any disturbance of another aircraft. To test stability of the entire system, we further experimented with both quadrocopters and the leader was flown in different shapes, orientations and with varying speeds.

\section{B. Shapes}

The leader follows waypoints along a given path produced by the iPad App. The operator can vary the size $s$, the speed $v$, and the type of the shape. Since every dataset includes a timestamp, the time $t$ since autonomy started can be calculated. We implemented waypoint navigation for a line along $\mathrm{x}$ or $\mathrm{y}$, circle and an eight-shape.

\section{Results}

In first experiments, we flew the AR.Drone manually along a straight path of $30 \mathrm{~m}$ back and forth and measured a position error of about $5 \%$ at the end. The floor was prepared to provide sufficient image features for the AR.Drone odometer using printed posters and thus we used the onboard odometry as ground truth data. For the focus of this work, tracking and following with another quadrocopter, the position error of the leader is not critical but only represents the performance of following the path of a given shape.

1) Only the Follower: In this experiment the follower did not hover in a stationary position but followed a small circular path of $80 \mathrm{~cm}$ in diameter in front of a fixed pattern, tracked by the NaturalPoint tracking system OptiTrack. Figure 5 and 6 show a section of the collected data of the tracking system and the onboard P3P pose estimation as well as the expected position.

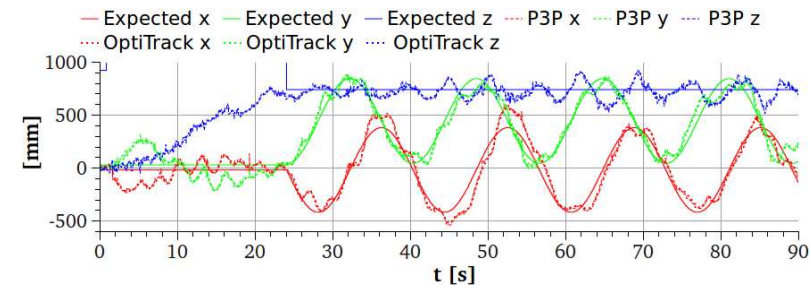

Fig. 5. The recorded path of the Hummingbird flying a circular path in front of a fixed pattern.

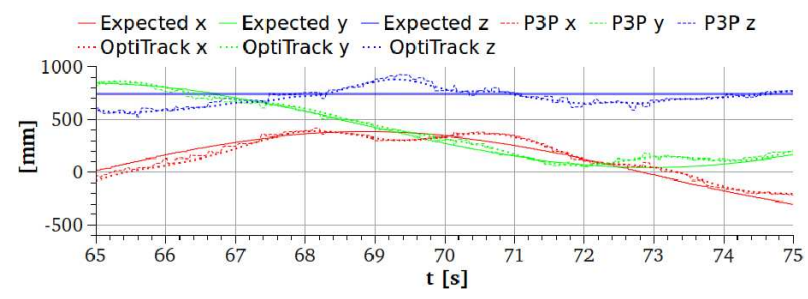

Fig. 6. Ten second clip of the recording shown in fig. 5.
TABLE I

Performance of our P3P Pose estimation AND THe PID CONTROLLER OF THE FOLLOWER. X, Y AND Z IN $\mathrm{cm}$

\begin{tabular}{|l||c|c|c|c|c|c|}
\hline \multicolumn{1}{|c||}{} & \multicolumn{3}{c|}{ P3P error } & \multicolumn{3}{c|}{ Flight accuracy } \\
& $\mathrm{X}$ & $\mathrm{Y}$ & $\mathrm{Z}$ & $\mathrm{X}$ & $\mathrm{Y}$ & $\mathrm{Z}$ \\
\hline Abs. Mean & 1.40 & 1.53 & 1.67 & 6.60 & 5.59 & 6.37 \\
Std. dev. & 1.26 & 1.33 & 1.51 & 4.71 & 4.21 & 4.46 \\
Max. err. & 13.83 & 11.82 & 12.93 & 24.3 & 19.70 & 24.0 \\
\hline
\end{tabular}

TABLE II

CONTROLLER CHARACTERISTICS OF AUTONOMOUS FLIGHTS OF A STRAIGHT-LINE PATH OF $1 \mathrm{M}$ LENGTH. X, Y AND Z IN $\mathrm{cm}, \psi \mathrm{IN}^{\circ}$

\begin{tabular}{|l||c|c|c|c|c|c|c|}
\hline \multicolumn{1}{|c||}{} & \multicolumn{3}{c|}{ Leader } & \multicolumn{4}{c|}{ Follower } \\
& $\mathrm{X}$ & $\mathrm{Y}$ & $\psi$ & $\mathrm{X}$ & $\mathrm{Y}$ & $\mathrm{Z}$ & $\psi$ \\
\hline Mean & 4.5 & -0.8 & 0.0 & 1.9 & -0.5 & 8.2 & -0.5 \\
Std. dev. & 26.2 & 27.8 & 0.3 & 17.4 & 25.0 & 18.3 & 7.5 \\
Max. err. & 90.8 & 97.0 & 1.1 & 68.4 & 68.4 & 61.5 & 34.3 \\
\hline
\end{tabular}

Table I shows statistics of the errors comparing the P3P pose estimation with the tracked pose of the tracking system during the whole experiment.

The absolute mean of less than $17 \mathrm{~mm}$ and the relatively small standard deviation for the pose estimation shows the stability of our P3P solution and allows the usage of this estimation as ground truth for experiments outside a tracking system, as is done for the following experiments. The standard deviation and the errors of the flight accuracy show uncritical oscillation around the desired position, considering the size of the quadrocopter $(53 \mathrm{~cm})$.

2) Straight Line: Table II shows the results of autonomous flights of both UAVs following a short straight-line path of $1 \mathrm{~m}$ length. The path was flown nine times with a given speed of $20 \mathrm{~cm} / \mathrm{s}$ in a small room. The short path results in a relatively harsh start, acceleration and stop sequence. The zerror of the AR.Drone is not given, because it is controlled by the aircraft standard hardware and not our controllers. Figure 7 shows the error (deviation to desired position) of the follower during this experiment.

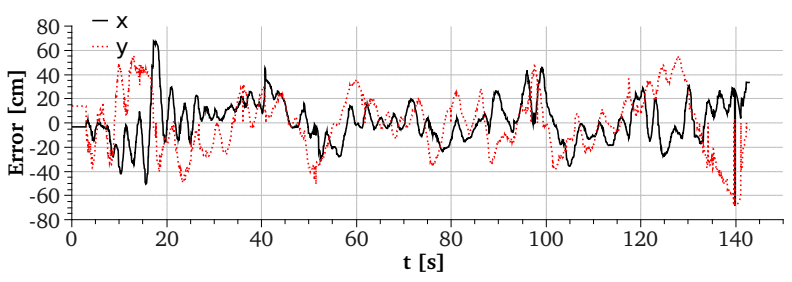

Fig. 7. Error of the follower flight of a short straight-line path of $1 \mathrm{~m}$ length repeated nine times.

Although the aircrafts were constantly moving, a standard deviaton of about $25 \mathrm{~cm}$ and a maximum error of about $1 \mathrm{~m}$ could be kept. This is sufficient for indoor flights in office buildings.

3) Circle: Ten successive autonomously flown circles with a diameter of $2 \mathrm{~m}$ at a given speed of $25 \mathrm{~cm} / \mathrm{s}$ were flown. The diameter equals the distance to the follower. An orthogonal orientation of the leader relative to the path 
TABLE III

CONTROLLER CHARACTERISTICS OF AUTONOMOUS FLIGHTS OF CIRCULAR PATH OF 2 M DIAMETER. X, Y AND Z IN $c m, \psi$ IN $^{\circ}$

\begin{tabular}{|l||c|c|c|c|c|c|c|}
\hline \multicolumn{1}{|c||}{} & \multicolumn{3}{c|}{ Leader } & \multicolumn{4}{c|}{ Follower } \\
& $\mathrm{X}$ & $\mathrm{Y}$ & $\psi$ & $\mathrm{X}$ & $\mathrm{Y}$ & $\mathrm{Z}$ & $\psi$ \\
\hline Mean & 0.6 & 0.6 & 14.4 & 0.6 & 1.3 & 13.5 & 0.1 \\
Std. dev. & 18.9 & 20.7 & 0.9 & 13.9 & 21.8 & 11.1 & 4.7 \\
Max. err. & 94.6 & 122.7 & 16.5 & 76.3 & 77.4 & 65.7 & 22.3 \\
\hline
\end{tabular}

TABLE IV

CONTROLLER CHARACTERISTICS OF MANUAL FLIGHT EXPERIMENTS IN AN EIGHT-SHAPE. X, Y AND Z IN $c m, \psi$ IN ${ }^{\circ}$

\begin{tabular}{|l||c|c|c|c|c|c|c|c|}
\hline \multicolumn{1}{|c||}{} & \multicolumn{4}{c|}{ Fast flight } & \multicolumn{4}{c|}{ Large flight } \\
& $\mathrm{X}$ & $\mathrm{Y}$ & $\mathrm{Z}$ & $\psi$ & $\mathrm{X}$ & $\mathrm{Y}$ & $\mathrm{Z}$ & $\psi$ \\
\hline Mean & 0.6 & 0.8 & 25.8 & 0.0 & 0.5 & 1.5 & 18.5 & 0.0 \\
S. dev. & 18.0 & 23.5 & 11.3 & 8.6 & 20.1 & 26.5 & 16.2 & 8.5 \\
M. err. & 95.8 & 79.4 & 64.8 & 34.5 & 67.6 & 95.1 & 72.1 & 34.5 \\
\hline
\end{tabular}

results in the follower flying on the same path at the opposite position. Table III shows the statistics of this experiment.

The quadrocopters flew slightly slower and the consistent sideway movement is smoother compared to the straight path experiments. This results in an even smaller standard deviation of the errors.

4) Figure-of-Eight: We flew multiple figures-of-eight in a sports hall with a manually flown leader until the battery of one of the aircrafts ran empty. We flew seven smaller eights, with a size of about $5 \mathrm{~m} \times 3 \mathrm{~m}$ at a relatively high speed without changing the leader orientation in a total of $160 \mathrm{~s}$ (Fig 8). Other three relatively large eights with a size of about $17 \mathrm{~m} \times 11 \mathrm{~m}$ were flown in $200 \mathrm{~s}$ but with consistently changing the leaders orientation. The statistics of both experiments are shown in Table IV.

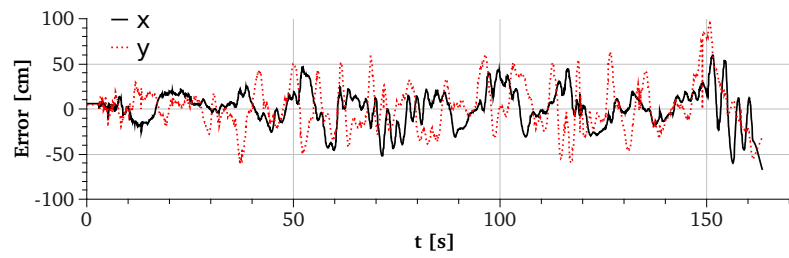

Fig. 8. Error of the follower of seven manually flown 8-shapes in $160 \mathrm{~s}$.

The standard deviation of about $25 \mathrm{~cm}$ and maximum error of below $1 \mathrm{~m}$ shows, that our follower provides comparable accuracy with a manually flown leader in a larger or faster, less accurate way.

\section{CONCLUSIONS AND FUTURE WORKS}

We have set up a follow-the-leader system with two heterogeneous quadrocopters. Our leader, a low-cost toy quadrocopter is capable of accurately flying autonomously along waypoints of a predefined path controlled by offboard PID-controllers. Another quadrocopter follows precisely only with additional low-cost hardware. We implemented an efficient solution of the P3P problem, effectively executing on an onboard microcontroller, enabling fast six degrees of freedom pose estimation. An update frequency of the follower of about $25 \mathrm{~Hz}$ is sufficient for precisely controlling the position but could be considerably improved by reducing the amount of data which is sent to the monitoring station. With a total standard deviation of below $35 \mathrm{~cm}$ for the leader and about $20 \mathrm{~cm}$ for the follower in $\mathrm{X}$ and $\mathrm{Y}$ and about $15 \mathrm{~cm}$ in $\mathrm{Z}$, both aircrafts are able to operate autonomously in narrow, GPS-denied indoor environments.

Disadvantages of our system are the artificial markers, which are required to localize the leader and the sensitivity against direct sunlight, which would preclude proper tracking of the pattern. Stronger LEDs would allow for a larger distance between leader and follower but also drain the batteries considerably faster. Tracking the leader with a standard camera would enable detection in the sun, but requires significantly more processing power. Onboard position control on the leader would eliminate the lag caused by WiFi communication and would allow for even more precise waypoint navigation.

\section{REFERENCES}

[1] R. W. Beard: Multiple UAV cooperative search under collision avoidance and limited range communication constraints. In IEEE Conference on Decision and Control (CDC), pages 25-30, 2003

[2] S. Bouabdallah, P. Murrieri, and R. Siegwart: Design and Control of an Indoor Micro Quadrotor. In IEEE International Conference on Robotics and Automation (ICRA), 2004

[3] A. K. Das, Student Member, R. Fierro, V. Kumar, Senior Member, J.P. Ostrowski, J. Spletzer and C. J. Taylor: A vision-based formation control framework. IEEE Transactions on Robotics and Automation, $18: 813-825,2002$

[4] M. A. Fischler and R. C. Bolles: Random sample consensus: a paradigm for model fitting with applications to image analysis and automated cartography. Commun. ACM, 24(6):381-395, 1981

[5] N. Guenard, T. Hamel: A practical visual servo control for an unmanned aerial vehicle. In: IEEE International Conference on Robotics and Automation (ICRA), pp. 1342-1348 2007

[6] B. Herisse, F.X. Russotto, T. Hamel, R.E. Mahony: Hovering fligh and vertical landing control of a VTOL unmanned aerial vehicle using optical flow. In: IEEE International Conference on Intelligent Robots and Systems (IROS), pp. 801-806 2008

[7] L. Kneip, D. Scaramuzza, R. Siegwart: A Novel Parametrization of the Perspective-Three-Point Problem for a Direct Computation of Absolute Camera Position and Orientation. In: Proc. of the 24th IEEE Conference on Computer Vision and Pattern Recognition (CVPR), 2011.

[8] A. Masselli, A. Zell: A Novel Marker Based Tracking Method for Position and Attitude Control of MAVs. In Proceedings of International Micro Air Vehicle Conference and Flight Competition (IMAV), 2012

[9] D. Mellinger, N. Michael, and V. Kumar: Trajectory generation and control for precise aggressive maneuvers with quadrotors. In Proceedings of the International Symposium on Experimental Robotics, 2010

[10] D. Mellinger, M. Shomin, N. Michael, and V. Kumar: Cooperative grasping and transport using multiple quadrotors. In Proceedings of the International Symposium on Distributed Autonomous Robotic Systems, 2010

[11] O.A.A. Orqueda and R. Fierro. A vision-based nonlinear decentralized controller for unmanned vehicles. In Robotics and Automation, 2006. ICRA 2006. Proceedings 2006 IEEE International Conference on, pages $1-6,2006$

[12] K.E. Wenzel, A. Zell: Automatic Take Off, Hovering and Landing Control for Miniature Helicopters with Low-Cost Onboard Hardware. In: Proceedings of AMS'09, Autonome Mobile Systeme 2009, pp. 7380. 2009

[13] K. E. Wenzel, A. Masselli, and A. Zell: Automatic Take Off, Tracking and Landing of a Miniature UAV on a Moving Carrier Vehicle. Journal of Intelligent \& Robotic Systems, 61:221-238, 2010

[14] B. Yun, B. Chen, K. Lum, and T. Lee: Design and implementation of a leader-follower cooperative control system for unmanned helicopters. Journal of Control Theory and Applications, 8:61-68, 2010 